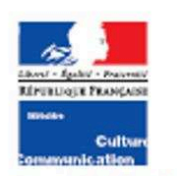

Secretarlat general Service de la

\title{
Peintres, graphistes, sculpteurs... Les artistes auteurs affiliés à la Maison des artistes en 2009
}

Marie GouYoN*
Painters, graphic designers, sculptors, etc. Artist-author members of the Maison des Artistes in 2009
En 2009, 24000 artistes auteurs sont affiliés à la Maison des artistes ${ }^{1}$. Ils se répartissent en douze disciplines, mais deux d'entre elles, le graphisme et la peinture, représentent $71 \%$ des effectifs (tableau 1). Près de 2000 illustrateurs ${ }^{2}$ sont affiliés à la Maison des artistes, 1800 sculp. teurs et 1300 artistes plasticiens.

La caractérisation des artistes auteurs et l'étude de l'évolution de la population depuis 1999 portent sur les seuls artistes affiliés à la Maison des artistes, auxquels ont été ajoutés les artistes assujettis qui, une année donnée, ont perçu des revenus supérieurs au seuil d'affiliation. La situation des assujettis n'a pas été approfondie ; quelques caractéristiques les concernant sont présentées en page 6.

Entre 1999 et 2007, le nombre d'artistes auteurs affiliés a augmenté en moyenne de $6 \%$ par an, passant ainsi de 13000 à 23000 ; depuis 2007, il est relativement stable (graphique 1).

Les progressions observées s'expliquent globalement par l'abaissement du seuil d'affiliation en $2001^{3}$ et par le développement de l'affiliation à titre dérogatoire, mais également par d'autres facteurs spécifiques à chaque discipline. Ainsi, les effectifs de graphistes affiliés ont été multipliés par 3,5 sur la période, jusqu’à dépasser ceux des
Tableau I - Répartition des artistes auteurs affiliés à la Maison des artistes selon la discipline en 2009

\begin{tabular}{|lcr|}
\hline Discipline* & Nombre & $\%$ \\
Graphiste & 9417 & 40 \\
Peintre & 7302 & 31 \\
Illustrateur & 1968 & 8 \\
Sculpteur & 1834 & 8 \\
Plasticien & 1285 & 5 \\
Dessinateur & 730 & 3 \\
Dessinateur textile & 547 & 2 \\
Graveur & 161 & 1 \\
Ceramiste & 63 & 0 \\
Vitrail & 53 & 0 \\
Decorateur & 30 & 0 \\
Tapissier & 9 & 0 \\
Ensemble & 23399 & 100 \\
Champ : auteurs amilas a la Malson des artistes en 2009, sur la base des \\
revenus odauteur percus en 2008. \\
-Nomanclature etable par la Malson des artistes. \\
\hline
\end{tabular}

Source: Maison des artistestorm: 2011

* Attachée statisticienne de l'Insee, chargée d’analyse au derps. Cette étude a bénéficié du concours actif de Pascal Murcins et Jean Corasu, respectivement chef du département des artistes et des professions à la Direction générale de la création artistique du ministère de la Culture et de la Communication, et adjoint de direction de la Maison des artistes. Qu' ils soient ici vivement remerciés. 1. L'affiliation est établie sur la base des revenus d'artiste auteur perçus en 2008 .

2. Illustrateurs exerçant principalement dans la presse et la publicité. Ľes illustrateurs dont les revenus d'auteur proviennent majoritairement de contrats d'édition relèvent de l'Agessa.

3. Le régime de l'affiliation repose sur la conversion d'un bénéfice non commercial artistique en l'équivalent d'un salaire permettant de valider 4 trimestres pour la retraite dans le régime général. Depuis 1993, ce seuil était fixé à 1200 fois la valeur horaire moyenne du Smic (vHMs); en 2001, il a été ramené à 900 visus. 


\section{Peintres, graphistes, sculpteurs... Les artistes auteurs affiliés à la Maison des artistes en 2009}

Painters, graphic designers, sculptors, etc. Artist-author members of the Maison des Artistes in 2009

Marie Gouyon

Éditeur : Département des études, de la prospective et des statistiques

Lieu d'édition : Paris

Année d'édition : 2011

Date de mise en ligne : 21 septembre 2015

Collection : Culture chiffres

ISBN électronique : 9782111398566

\section{Sbooks}

http://books.openedition.org

\section{Édition imprimée}

Date de publication : 1 avril 2011

Nombre de pages : 8

\section{Référence électronique}

GOUYON, Marie. Peintres, graphistes, sculpteurs... Les artistes auteurs affiliés à la Maison des artistes en 2009. Nouvelle édition [en ligne]. Paris : Département des études, de la prospective et des statistiques, 2011 (généré le 25 avril 2021). Disponible sur Internet : <http://books.openedition.org/deps/528>.

ISBN : 9782111398566. 

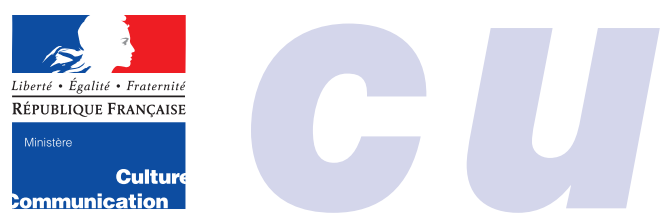

Secrétariat général

Service de la

coordination des

politiques culturelles

et de l'innovation

Département

des études,

de la prospective

et des statistiques

\section{Peintres, graphistes, sculpteurs... Les artistes auteurs affiliés à la Maison des artistes en 2009}

\section{Marie Gouyon* \\ Painters, graphic designers, sculptors, etc. Artist-author members of the Maison des Artistes in 2009}

En 2009, 24000 artistes auteurs sont affiliés à la Maison des artistes ${ }^{1}$. Ils se répartissent en douze disciplines, mais deux d'entre elles, le graphisme et la peinture, représentent $71 \%$ des effectifs (tableau 1). Près de 2000 illustrateurs $^{2}$ sont affiliés à la Maison des artistes, 1800 sculpteurs et 1300 artistes plasticiens.

La caractérisation des artistes auteurs et l'étude de l'évolution de la population depuis 1999 portent sur les seuls artistes affiliés à la Maison des artistes, auxquels ont été ajoutés les artistes assujettis qui, une année donnée, ont perçu des revenus supérieurs au seuil d'affiliation. La situation des assujettis n'a pas été approfondie ; quelques caractéristiques les concernant sont présentées en page 6 .

Entre 1999 et 2007, le nombre d'artistes auteurs affiliés a augmenté en moyenne de $6 \%$ par an, passant ainsi de 13000 à 23000 ; depuis 2007, il est relativement stable (graphique 1).

Les progressions observées s'expliquent globalement par l'abaissement du seuil d'affiliation en $2001^{3}$ et par le développement de l'affiliation à titre dérogatoire, mais également par d'autres facteurs spécifiques à chaque discipline. Ainsi, les effectifs de graphistes affiliés ont été multipliés par 3,5 sur la période, jusqu'à dépasser ceux des

\section{Tableau 1 - Répartition des artistes auteurs affiliés à la Maison des artistes selon la discipline en 2009}

\begin{tabular}{|lcr|}
\hline Discipline* & Nombre & $\%$ \\
Graphiste & 9417 & 40 \\
Peintre & 7302 & 31 \\
Illustrateur & 1968 & 8 \\
Sculpteur & 1834 & 8 \\
Plasticien & 1285 & 5 \\
Dessinateur & 730 & 3 \\
Dessinateur textile & 547 & 2 \\
Graveur & 161 & 1 \\
Céramiste & 63 & 0 \\
Vitrail & 53 & 0 \\
Décorateur & 30 & 0 \\
Tapissier & 9 & 0 \\
Ensemble & $\mathbf{2 3} 399$ & 100 \\
& & \\
Champ: auteurs affiliés à la Maison des artistes en 2009, sur la base des \\
revenus d'auteur perçus en 2008. \\
*Nomenclature établie par la Maison des artistes. \\
\hline
\end{tabular}

Source : Maison des artistes/DEPS, 2011

\footnotetext{
* Attachée statisticienne de l'Insee, chargée d'analyse au DEPS. Cette étude a bénéficié du concours actif de Pascal MuRGIER et Jean CoRBU, respectivement chef du département des artistes et des professions à la Direction générale de la création artistique du ministère de la Culture et de la Communication, et adjoint de direction de la Maison des artistes. Qu'ils soient ici vivement remerciés.

1. L'affiliation est établie sur la base des revenus d'artiste auteur perçus en 2008.

2. Illustrateurs exerçant principalement dans la presse et la publicité. Les illustrateurs dont les revenus d'auteur proviennent majoritairement de contrats d'édition relèvent de l'Agessa.

3. Le régime de l'affiliation repose sur la conversion d'un bénéfice non commercial artistique en l'équivalent d'un salaire permettant de valider 4 trimestres pour la retraite dans le régime général. Depuis 1993, ce seuil était fixé à 1200 fois la valeur horaire moyenne du Smic (VHMS); en 2001, il a été ramené à 900 vHMS.
} 


\section{Le régime de sécurité sociale des artistes auteurs affiliés}

Toute diffusion ou exploitation commerciale, par un diffuseur ${ }^{1}$, d'une œuvre originale d'un artiste auteur donne lieu au versement d'une rémunération (les droits d'auteur). L'acquisition de l'œuvre (tableau, sculpture, etc.) donne lieu également à rémunération. Lorsque l'artiste auteur exerce son activité de façon indépendante (non salariée) et qu'il réside fiscalement en France, le versement des droits d'auteur par le diffuseur ou la vente d'œuvres s'accompagnent du prélèvement de cotisations et contributions obligatoires, opération appelée précompte. Ces cotisations et contributions sont collectées par deux associations agréées:

- la Maison des artistes pour les œuvres d'arts graphiques et plastiques:

- l'Association pour la gestion de la sécurité sociale des auteurs (Agessa) pour les activités de création littéraire, dramatique, musicale, audiovisuelle et photographique.

Les cotisations aux assurances sociales (vieillesse, maladie, maternité, invalidité, décès), la CSG et la CRDS sont dues par l'artiste auteur ${ }^{2}$ au titre du régime de protection sociale des artistes auteurs (articles L.382-1, R.382-1 et suivants du Code de la sécurité sociale); ce régime spécifique est rattaché au régime général des salariés.

Sous conditions de ressources et s'il en fait la demande, l'artiste auteur est affilié à la Maison des artistes ou à l'Agessa ; à ce titre, il bénéficie des prestations équivalentes à celles du régime général (indemnités journalières, congés maternité, etc.). Pour cela, il doit avoir perçu, l'année civile qui précède, des revenus d'auteur ${ }^{3}$ supérieurs au seuil d'affiliation, soit 900 fois la valeur du Smic horaire (7524 euros de revenus sur l'année 2007, 7749 euros de revenus sur l'année 2008). Lorsque le seuil d'affiliation n'est pas atteint, l'affiliation ou son maintien peut être prononcé, à titre dérogatoire, par la commission professionnelle de la Maison des artistes ou de l'Agessa, qui juge de l'engagement professionnel de l'artiste auteur. S'il n'est pas affilié, il est dit assujetti.

1. Par exemple, les galeries d'art, les éditeurs, les producteurs de films. Les sociétés d'auteurs (telles que la Sacem, la Scam, la SACD) reversent à l'Agessa les cotisations afférentes aux droits d'auteur qu'elles répartissent. 2. Le diffuseur quant à lui verse à l'Agessa ou à la Maison des artistes une contribution représentant $1 \%$ de la rémunération versée, $1 \%$ des commissions perçues (sociétés de ventes volontaires) ou $1 \%$ de $30 \%$ du chiffre d'affaires issu de la vente d'œuvres artistiques.

3 . On entend par revenus d'auteur les revenus imposables au titre des bénéfices non commerciaux majorés de $15 \%$ ou les droits d'auteur bruts s'ils sont déclarés en traitements et salaires.

peintres en 2007. Cet essor traduit à la fois le développement général des activités liées au graphisme, mais aussi l'affiliation récente (2006) à la Maison des artistes de graphistes cotisant auparavant au régime général des salariés ou au régime des travailleurs indépendants. Le nombre de plasticiens quant à lui a triplé en dix ans, traduisant le développement des nouvelles formes de création dans ce domaine (installations, vidéos, œuvres éphémères, etc.).

Les effectifs de sculpteurs et de peintres se sont élevés respectivement de $35 \%$ et de $27 \%$ entre 1999 et 2008, puis ont diminué de $4 \%$ et $6 \%$ au cours de la dernière année.
Graphique 1 - Affiliés à la Maison des artistes selon la discipline, 1999-2009

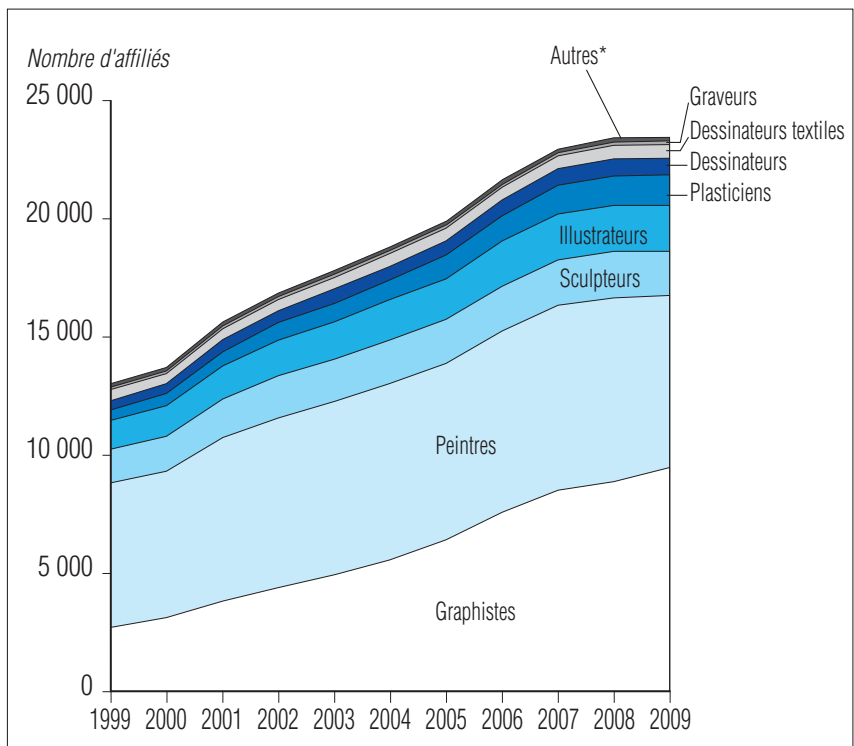

Champ : ensemble des artistes auteurs affiliés à la Maison des artistes entre 1999 et 2009, sur la base des revenus d'auteur perçus l'année précédente. * Autres : artistes céramistes, du vitrail, du décor ou de la tapisserie. Note de lecture : 12959 artistes étaient affiliés en 1999 (dont 2678 graphistes) et 23399 en 2009 (dont 9417 graphistes).

Source : Maison des artistes/DEPS, 2011

\section{Des femmes plus nombreuses mais toujours minoritaires}

Les hommes représentent $58 \%$ des artistes affiliés à la Maison des artistes, contre $53 \%$ de l'ensemble des personnes occupant un emploi en France ${ }^{4}$. Ils sont fortement majoritaires dans la plupart des disciplines et en premier lieu parmi les sculpteurs (68\%), les illustrateurs (63\%), les peintres $(61 \%)$ et les dessinateurs $(61 \%)$. Les femmes sont majoritaires dans la céramique et le vitrail. Une seule discipline est presque entièrement féminine, celle des designers textiles, où les femmes représentent $88 \%$ des affiliés.

Depuis 1999, la part des femmes affiliées à la Maison des artistes progresse, passant de $33 \%$ à $42 \%$. Cette progression est particulièrement marquée parmi les plasticiens, les peintres et les graveurs, disciplines auparavant les moins féminisées. En revanche, leur représentation parmi les graphistes a relativement moins progressé et leur part est restée la même parmi les dessinateurs (graphique 2).

\section{Une population de moins en moins âgée du fait du développement des disciplines jeunes}

Les artistes affiliés à la Maison des artistes sont âgés en moyenne de 44 ans, soit trois ans de plus que l'âge moyen des actifs occupant un emploi ${ }^{5}$ en 2008.

4. D'après l'enquête Emploi 2008 de l'Insee.

5. D'après l'enquête Emploi 2008 de l'Insee. Les écrivains, photographes, compositeurs et autres artistes affiliés à l'Agessa en 2008 sont âgés en moyenne de 49 ans (Agessa/DEPS, 2011). 
Graphique 2 - Les femmes parmi les auteurs affiliés à la Maison des artistes selon la discipline, 1999, 2004 et 2009

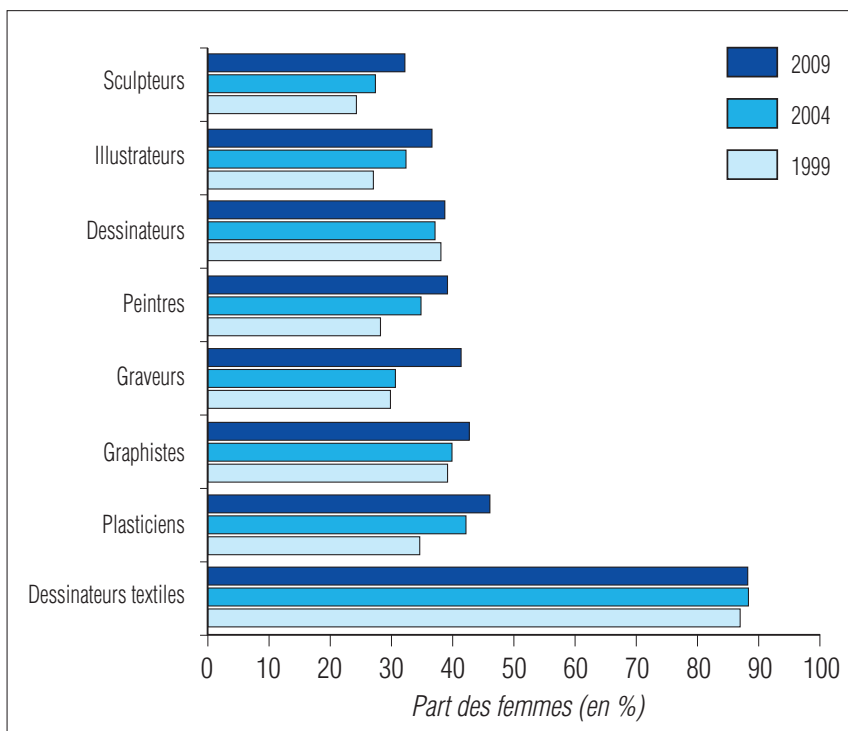

Champ : ensemble des artistes auteurs affiliés à la Maison des artistes en 1999, 2004 et 2009, sur la base des revenus d'auteur perçus en 1998, 2003 et 2008. Les disciplines de faibles effectifs (décoration, céramique, vitrail et tapisserie) ne sont pas représentées ici.

Note de lecture : les femmes représentaient $35 \%$ des plasticiens en 1999 , $42 \%$ en 2004 et $46 \%$ en 2009 .

Source : Maison des artistes/DEPS, 2011

Certaines disciplines sont jeunes, en particulier le graphisme. Dans ce domaine, les affiliés ont en moyenne 38 ans en 2009 et un affilié sur cinq a moins de 30 ans. L'afflux de jeunes graphistes entre 1999 et 2004 a contribué à rajeunir la discipline. L'illustration, le dessin (y compris textile) et les arts plastiques sont également des disciplines assez jeunes, l'âge moyen y avoisine 42 ans. Cette moyenne se maintient depuis une dizaine d'années pour les illustrateurs et les plasticiens. En revanche, la population des dessinateurs a nettement rajeuni (ils avaient en moyenne 47 ans en 1999), notamment grâce à l'entrée sur le marché de jeunes artistes (la part des moins de 30 ans est ainsi passée de $3 \%$ à $11 \%$ ). À l'inverse, la part des moins de 40 ans parmi les dessinateurs textiles s'est fortement contractée, passant de $69 \%$ en 1999 à $42 \%$ en 2009, élevant l'âge moyen de ces affiliés de 38 ans en 1999 à 42 ans dix ans plus tard.

Dans la gravure, la peinture et la sculpture, les artistes affiliés sont beaucoup plus âgés. Les graveurs et les peintres ont, en moyenne, respectivement 49 ans et 51 ans, en 2009 comme en 1999. La population des sculpteurs, quant à elle, a vieilli au cours de la période, l'âge moyen des artistes y passant de 49 à 52 ans; en 2009, un sculpteur sur cinq a plus de 60 ans (graphique 3 ).
Graphique 3 - Âge moyen des artistes auteurs affiliés à la Maison des artistes selon la discipline, 1999, 2004 et 2009

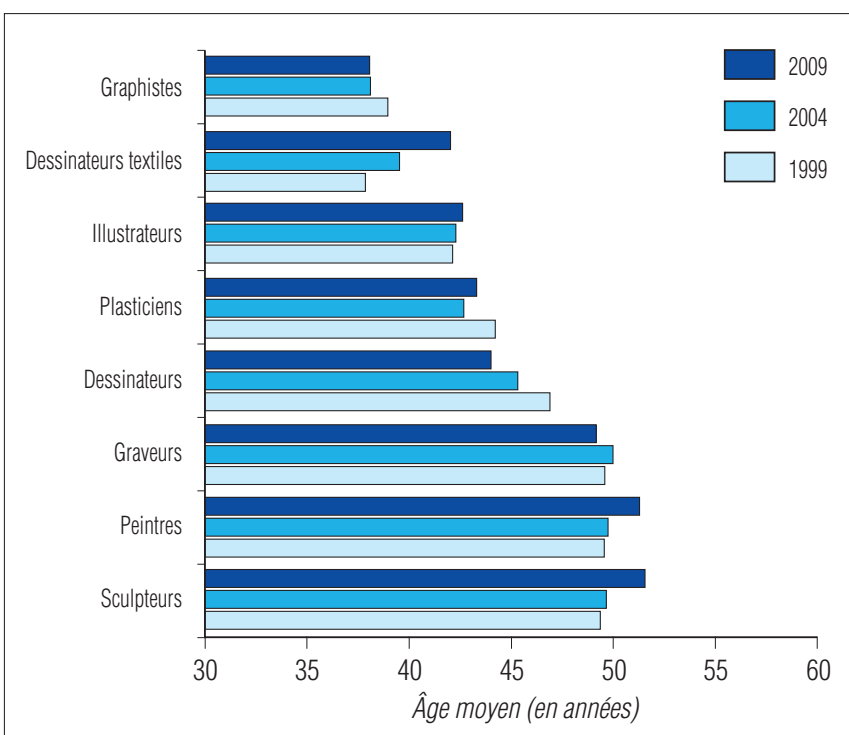

Champ : ensemble des artistes auteurs affiliés à la Maison des artistes en 1999, 2004 et 2009, sur la base des revenus d'auteur perçus en 1998, 2003 et 2008. Les disciplines de faibles effectifs (décoration, céramique, vitrail et tapisserie) ne sont pas représentées ici.

Note de lecture: en 1999, les dessinateurs textiles ont en moyenne 38 ans, 40 ans en 2004 et 42 ans en 2009.

Source : Maison des artistes/DEPS, 2011

\section{De longue date, une population très concentrée en Île-de-France}

Un tiers des artistes affiliés en 2009 à la Maison des artistes habitent Paris intra-muros et plus de la moitié en Île-de-France ${ }^{6}$. La surreprésentation des franciliens varie de $40 \%$ à $70 \%$ selon les disciplines : elle s'établit à $68 \%$ parmi les dessinateurs textiles, environ $60 \%$ pour les graphistes, illustrateurs et dessinateurs. Autour de la moitié des plasticiens, graveurs et peintres et $36 \%$ des sculpteurs résident en Île-de-France (graphique 4).

La prépondérance de l'Île-de-France, en termes d'effectifs d'artistes, est globalement stable (54\% en 1999 comme en 2009). Toutefois, elle tend à se réduire pour les plasticiens, les sculpteurs et surtout pour les peintres. Elle s'accentue en revanche parmi les dessinateurs, plus nombreux à vivre en Île-de-France en 2009 qu'en 1999 (+ 5 points). Pour ces derniers comme pour les dessinateurs textiles, Paris gagne du terrain par rapport au reste de l'île-deFrance.

6. En 2008, $21 \%$ des personnes occupant un emploi en France vivent dans la région parisienne, selon l'enquête Emploi de l'Insee. Les écrivains, photographes, compositeurs et autres artistes auteurs affiliés à l'Agessa en 2008 sont $38 \%$ à vivre à Paris intra-muros et $61 \%$ en Île-de-France (Agessa/DEPS). 


\section{Graphique 4-Répartition géographique des auteurs affiliés à la Maison des artistes selon la discipline, 1999-2009}

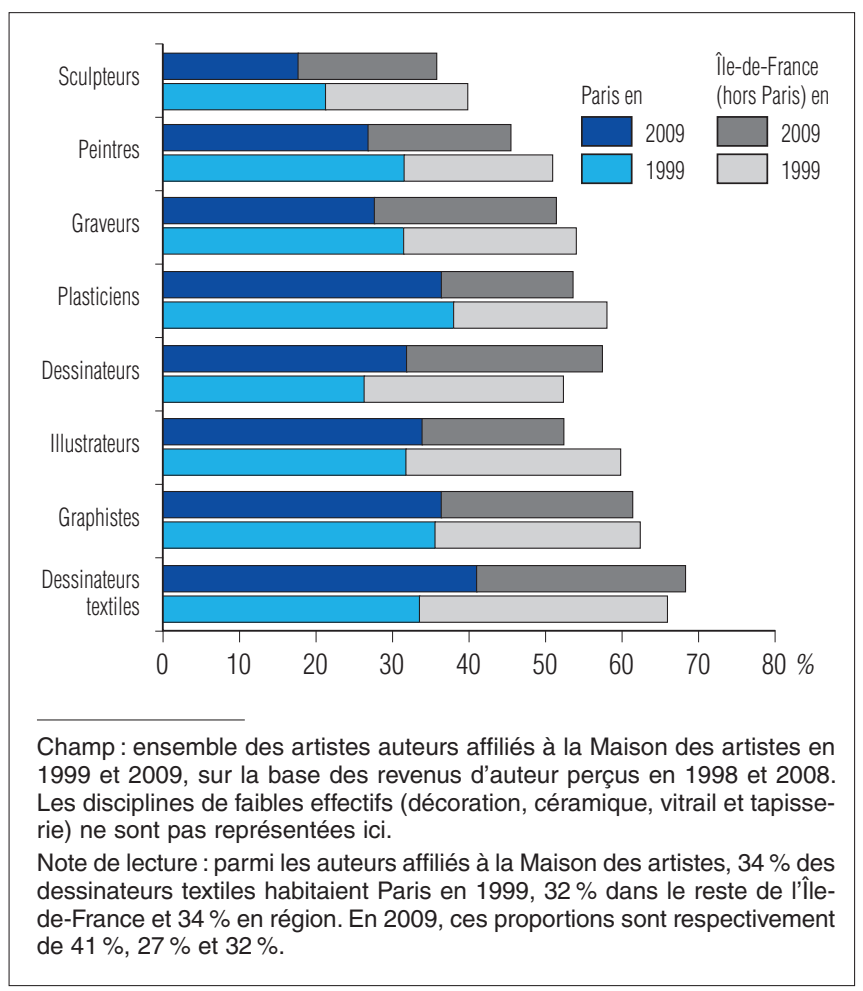

Source : Maison des artistes/DEPS, 201

\section{Des revenus artistiques ${ }^{7}$ très dispersés}

Les revenus artistiques moyens des affiliés à la Maison des artistes atteignent, en moyenne, près de 24000 euros de bénéfices non commerciaux en $2009^{8}$. Cette moyenne recouvre de très fortes disparités d'une discipline à l'autre : le revenu annuel moyen issu des activités artistiques des graphistes, illustrateurs et dessinateurs textiles avoisine 27000 euros en 2009 tandis que celui des sculpteurs et des graveurs est inférieur à 20000 euros (graphique 5).

Cependant, au sein d'une même discipline, les revenus artistiques sont très variables d'un auteur à l'autre, une minorité percevant des revenus très élevés tandis que la majorité déclare des revenus assez bas. Ainsi, selon les disciplines, le revenu médian est de $30 \%$ à $50 \%$ inférieur au revenu moyen.

En 2009, la moitié des artistes déclarent avoir perçu l'année précédente moins de 13700 euros annuels de bénéfices non commerciaux (BNC). À titre de comparaison, la moitié des salariés travaillant à temps plein dans le privé et le semi-public ont perçu en 2008 un salaire annuel net inférieur à 18400 euros, soit $34 \%$ de plus ${ }^{9}$.

$\mathrm{Au}$ cours de la décennie passée, toutes disciplines confondues, les revenus artistiques ${ }^{10}$ ont augmenté de $7 \%$ en médiane et de $3 \%$ en moyenne (en euros constants). S'ils se sont effectivement élevés pour les graveurs, les plasticiens et les dessinateurs textiles, ils ont diminué pour les gra-

\section{Graphique 5 - Répartition des revenus d'auteur perçus par les artistes auteurs affiliés à la Maison des artistes}

selon la discipline, 1998-2008

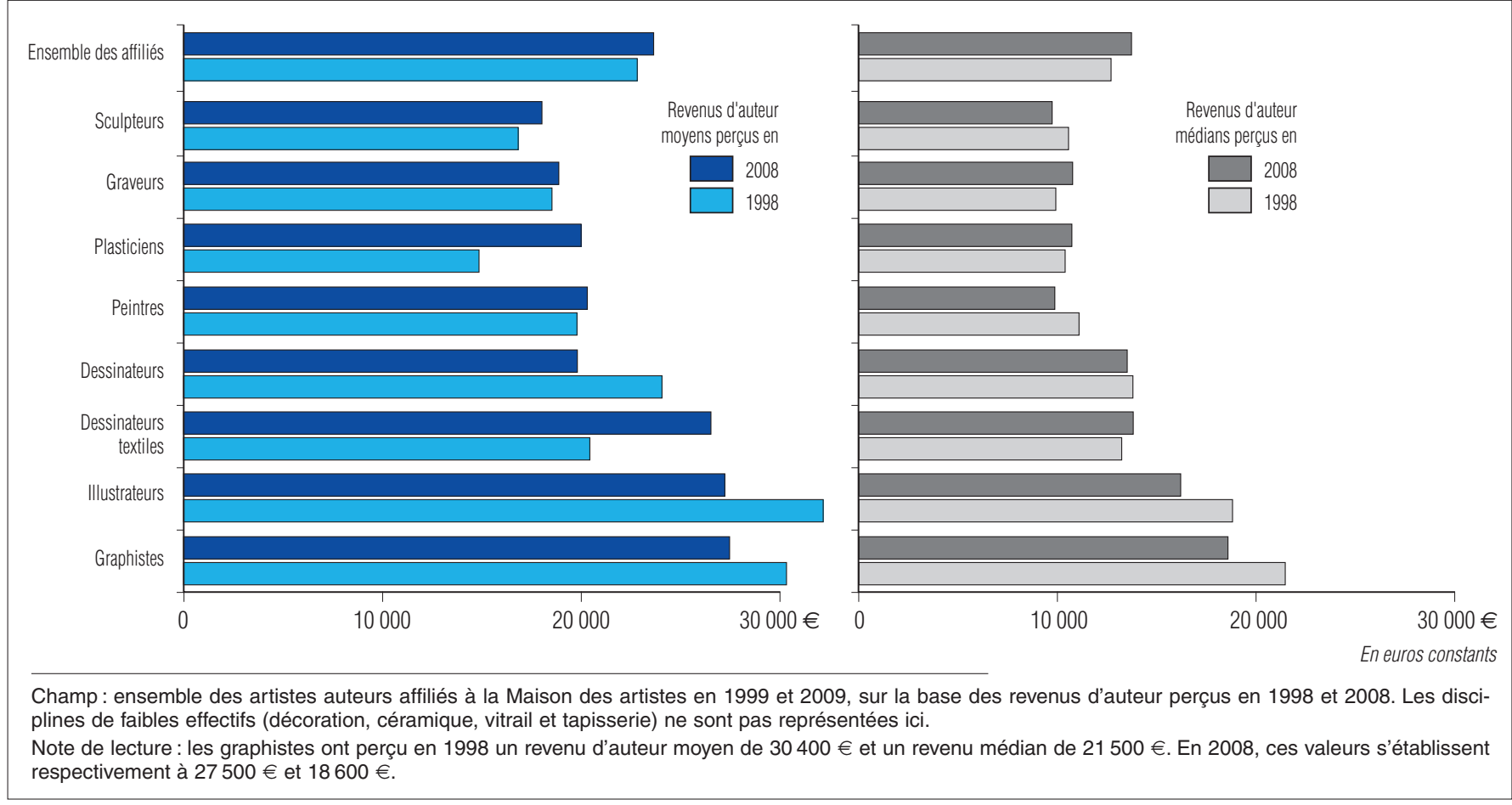

Source : Maison des artistes/DEPS, 201

7. La source exploitée couvre uniquement les revenus issus des activités artistiques et ne tient pas compte des éventuelles autres sources de revenus des affiliés : elle ne permet donc pas de mesurer l'ensemble des revenus perçus par les artistes auteurs ni de refléter leur niveau de vie.

8. Les peintres, sculpteurs, graveurs et plasticiens tirent l'essentiel de leurs revenus d'auteur de la vente de leurs œuvres, tandis que les graphistes et les dessinateurs textiles perçoivent des revenus liés à l'exploitation de leurs œuvres. À titre de comparaison, les artistes auteurs affiliés à l'Agessa déclarent en 2008 près de 40000 euros en moyenne en traitements et salaires et 29000 euros en bénéfices non commerciaux.

9. Source : Déclaration annuelle des données sociales (DADS) 2008, Insee.

10. Revenus d'auteur de 1998 et 2008 perçus par les artistes auteurs affiliés en 1999 et 2009. 


\section{Graphique 6 - Concentration des bénéfices non commerciaux en 2009}

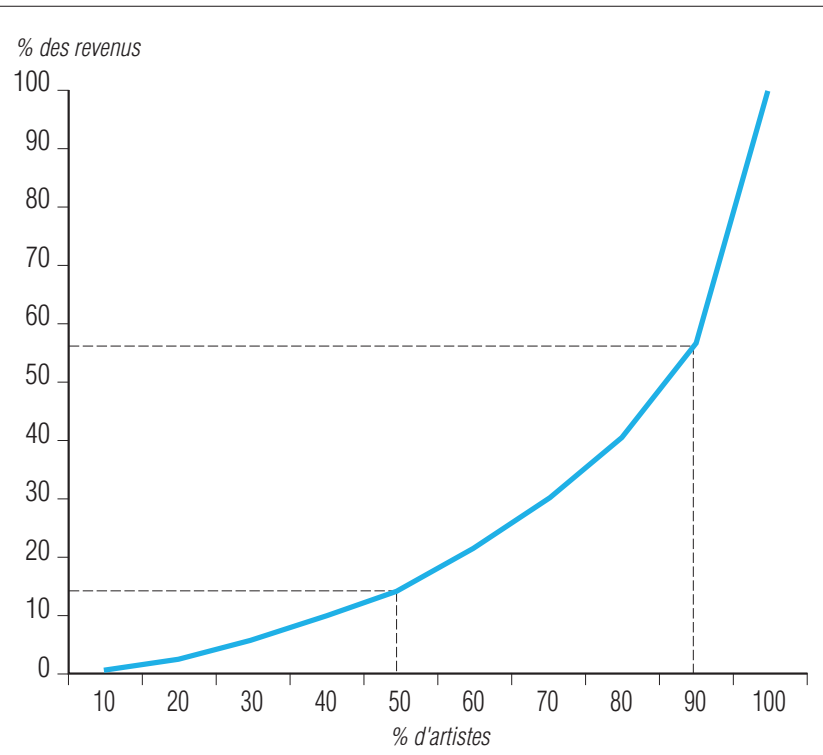

Champ : ensemble des artistes auteurs affiliés à la Maison des artistes en 2009, sur la base des revenus d'auteur perçus en 2008.

Note de lecture: en 2009, $50 \%$ des artistes déclarent avoir perçu l'année précédente $15 \%$ de l'ensemble des revenus d'auteur, et les $10 \%$ percevant les plus hauts revenus, $43 \%$.

Source : Maison des artistes/DEPS, 201

phistes, illustrateurs et dessinateurs. Rappelons que ces derniers œuvrent dans des disciplines qui se sont considérablement développées en dix ans, les effectifs des graphistes ayant plus que triplé, et ceux des illustrateurs et des dessinateurs s'étant élevés respectivement de plus de moitié.

Conformément à une caractéristique répandue parmi les professions artistiques, les revenus artistiques sont très concentrés : la moitié des auteurs cumulent $15 \%$ des revenus, tandis que les $10 \%$ d'artistes les mieux rémunérés en captent à eux seuls $43 \%$ (graphique 6).

Ce phénomène est à la fois très stable au cours des dix dernières années et caractéristique de toutes les disciplines

\section{Graphique 7 - Part des revenus des $10 \%$ d'artistes auteurs affiliés les mieux rémunérés selon la discipline, 1998-2008}

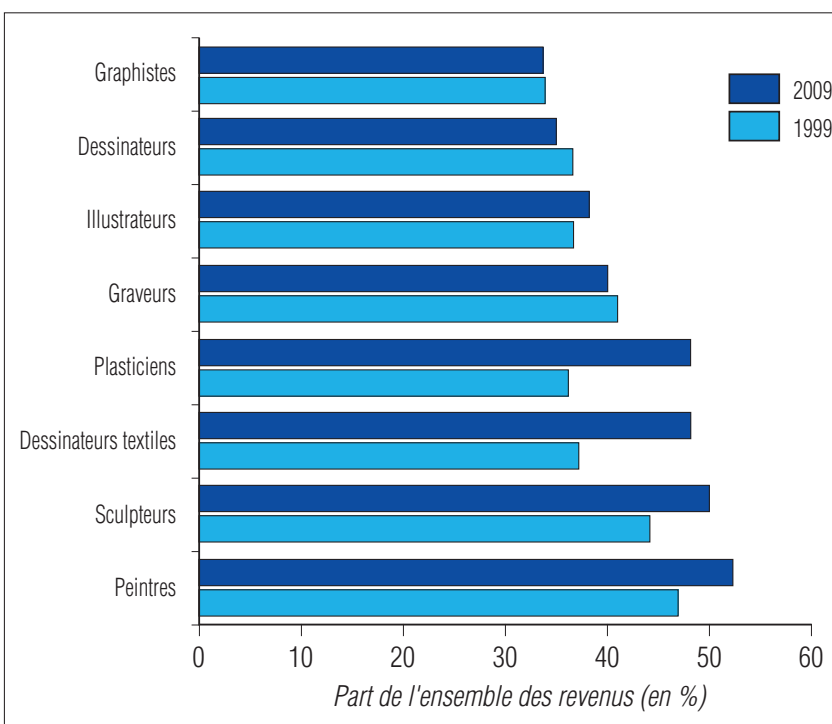

Champ : ensemble des artistes auteurs affiliés à la Maison des artistes en 1999 et 2009, sur la base des revenus d'auteur percus en 1998 et 2008 . Les disciplines de faibles effectifs (décoration, céramique, vitrail et tapisserie) ne sont pas représentées ici.

Note de lecture : en 2009, parmi les peintres, les $10 \%$ d'artistes les mieux rémunérés se partagent $52 \%$ de l'ensemble des revenus perçus en 2008 dans la discipline; cette proportion était de $47 \%$ en 1999.

Source : Maison des artistes/DEPS, 2011

plastiques et graphiques, dans des proportions variables. Ainsi, les revenus issus des activités du graphisme et du dessin sont nettement moins dispersés que ceux des peintres et des plasticiens : les $10 \%$ d'artistes percevant les plus hauts revenus captent un tiers des bénéfices non commerciaux des graphistes et des dessinateurs, et plus de la moitié des revenus des peintres et des sculpteurs. Pour les dessinateurs et les plasticiens, la part de l'ensemble des revenus captés par les artistes les plus favorisés s'est étoffée en dix ans : les $10 \%$ d'artistes les mieux rémunérés se partageaient $36 \%$ des revenus de ces disciplines à la fin des années 1990, 48 \% dix ans plus tard (graphique 7). 


\section{Assujettis : \\ les artistes auteurs qui cotisent à la Maison des artistes sans y être affiliés}

Les assujettis (c'est-à-dire les cotisants à la Maison des artistes n'atteignant pas le seuil d'affiliation, qui n'ont pas demandé, n'ont pas obtenu leur affiliation à titre dérogatoire ou encore dont l'affiliation n'a pas été maintenue faute de revenus d'auteur suffisants) ne bénéficient pas du régime de sécurité sociale des artistes auteurs. Ils sont devenus de plus en plus nombreux tout au long de la période jusqu'à devenir presque aussi nombreux que les affiliés à la Maison des artistes: ils représentaient en effet $18 \%$ du total des cotisants en 1999, $33 \%$ en 2004 et $47 \%$ en 2009 (graphique A).

Le nombre d'artistes assujettis dans les différentes disciplines a été multiplié par au moins 5 en dix ans, et même par 14 pour les plasticiens et par 16 pour les graphistes.

Si les artistes affiliés à la Maison des artistes sont majoritairement des hommes, la situation inverse prévaut parmi les assujettis: $54 \%$ des cotisants qui ne bénéficient pas de la sécurité sociale des artistes auteurs sont des femmes (tableau A). Elles représentent $92 \%$ des dessinateurs textiles, $61 \%$ des plasticiens, $60 \%$ des graveurs et $56 \%$ des peintres assujettis. Au sein de la population des assujettis, parmi les graphistes, les illustrateurs, les dessinateurs et les sculpteurs, les femmes sont pratiquement aussi nombreuses que les hommes.

La population des assujettis est plus jeune que celle des affiliés à la Maison des artistes : $47 \%$ des premiers ont moins de 40 ans, $42 \%$ parmi les seconds. L'écart est particulièrement saillant dans l'illustration et le dessin (y compris textile).

Les assujettis résident moins souvent en Île-de-France (43\%) que les affiliés (54\%), d'autant moins qu'ils sont plasticiens ou dessinateurs.

\section{Des revenus en moyenne faibles et dispersés}

Selon la discipline, les assujettis à la Maison des artistes déclarent en 2009 en moyenne entre 1950 et 2400 euros. Toutefois, comme pour les affiliés, le revenu artistique moyen des assujettis recouvre une réalité plus contrastée. Les $10 \%$ de graphistes les mieux rémunérés déclarent plus de 5515 euros (et moins de 7749 euros : au-delà de ce seuil, les artistes auteurs sont affiliés à la Maison des artistes). Les plasticiens assujettis les plus favori-

\section{Tableau A - Caractéristiques sociodémographiques et revenus perçus en 1998 et en 2008}

par les artistes assujettis et affiliés à la Maison des artistes (données 2009)

En \% et en euros constants

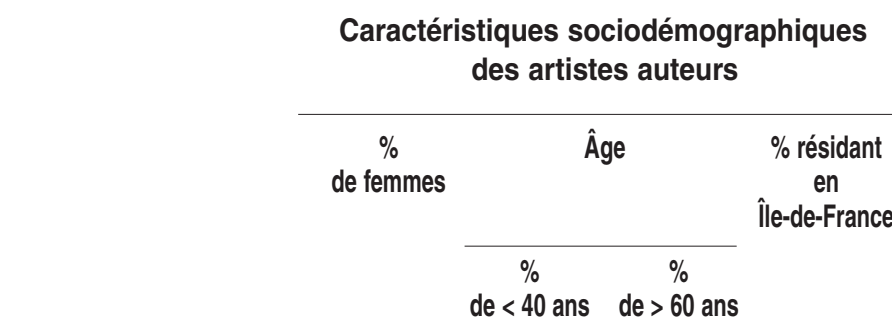

Revenus d'auteur perçus par les artistes auteurs en...

\begin{tabular}{|c|c|c|c|}
\hline \multicolumn{2}{|r|}{1998} & \multicolumn{2}{|r|}{2008} \\
\hline Moyens & $\begin{array}{l}\text { Les } 10 \% \text { percevant } \\
\text { les revenus les plus } \\
\text { élevés déclarent... }\end{array}$ & Moyens & $\begin{array}{l}\text { Les } 10 \% \text { percevant } \\
\text { les revenus les plus } \\
\text { élevés déclarent... }\end{array}$ \\
\hline$e n €^{*}$ & $\begin{array}{l}\% \text { du } \quad+\text { de... } \\
\text { total } \\
\text { en } €^{*}\end{array}$ & $e n €^{*}$ & $\begin{array}{l}+ \text { de... } \\
e n €^{*}\end{array}$ \\
\hline
\end{tabular}

\section{Assujettis}

Graphiste

Peintre

Illustrateur

Sculpteur

Plasticien

Dessinateur

Dessinateur textile

Graveur

$\begin{array}{ll}48 & 79 \\ 56 & 25 \\ 49 & 70 \\ 48 & 23 \\ 61 & 52 \\ 48 & 59 \\ 92 & 62 \\ 60 & 37\end{array}$

3
25
8
22
6
12
5
19

52
38
50
34
38
47
63
51

3085
2904
3251
2459
2913
3128
3332
3015
$\mathbf{2 9 0 8}$

$\begin{array}{lllll}24 & 6759 & 2385 & 27 & 5515 \\ 26 & 6857 & 1952 & 29 & 4450 \\ 23 & 7309 & 2239 & 28 & 5035 \\ 28 & 5660 & 1954 & 28 & 4401 \\ 26 & 6963 & 1938 & 29 & 4392 \\ 24 & 6951 & 2235 & 29 & 5397 \\ 22 & 7320 & 2053 & 28 & 4583 \\ 25 & 6494 & 2102 & 26 & 4581 \\ \mathbf{2 6} & \mathbf{6 7 8 8} & \mathbf{2 1 0 4} & \mathbf{2 9} & \mathbf{4 8 0 9}\end{array}$

Ensemble

54

47

14

43

2908

30388

19839

32202

16903

14909

24118

20520

18544

22885
$-19-57562$

$18 \quad 36082$

$23 \quad 75292$

$18 \quad 31245$

$21 \quad 31052$

$23 \quad 55334$

$21 \quad 43404$

$26 \quad 47664$

27491

$21 \quad 59083$

20333

27268

18077

20038

19818

26574

18874

$18 \quad 37593$

$24 \quad 65126$

$18 \quad 32471$

$20 \quad 40326$

$22 \quad 42857$

$18 \quad 48746$

$23 \quad 44143$

Ensemble

42

10

54

$22 \quad 49469 \quad 23682$

$20 \quad 48395$

Champ : ensemble des artistes auteurs assujettis d'une part, affiliés d'autre part à la Maison des artistes en 1999 et 2009 (sur la base des revenus d'auteur perçus en 1998 et 2008).

Note de lecture: en 2009, parmi les graphistes, les femmes représentent $48 \%$ des assujettis. En 2008 , les graphistes assujettis ont perçu en moyenne 2385 euros en bénéfices non commerciaux; les $10 \%$ de graphistes assujettis les mieux rémunérés ont déclaré entre 5515 euros et 7749 euros (cette dernière somme correspondant au seuil d'affiliation) et se sont partagé $27 \%$ de l'ensemble des revenus des graphistes assujettis.

* Euros constants. 
Graphique A - Affiliés et assujettis à la Maison des artistes, 1999-2009

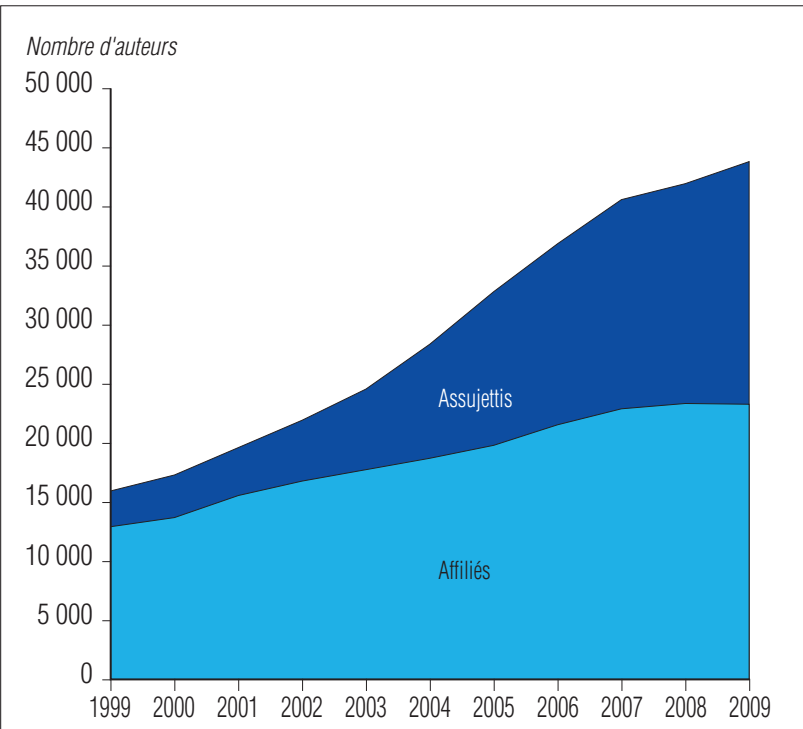

Champ : ensemble des artistes auteurs affiliés et assujettis à la Maison des artistes, de 1999 à 2009 (sur la base des revenus d'auteur perçus l'année précédente).

Note de lecture: en 1999, la Maison des artistes dénombre 12959 artistes affiliés et 2922 assujettis ; en 2009, 23399 sont affiliés à l'organisme et 20348 y sont assujettis. sés perçoivent, eux, entre 4400 et 7750 euros. Quelle que soit la discipline, les $10 \%$ d'artistes les mieux rémunérés perçoivent autour de $27 \%$ de l'ensemble des revenus artistiques de la discipline.

Les revenus artistiques des assujettis ont nettement diminué depuis la fin des années 1990. L'abaissement de $25 \%$ du seuil d'affiliation intervenu en 2001 explique en partie ce phénomène : les assujettis dont les revenus d'auteurs se situaient en deçà du seuil se sont vus affiliés. Par ailleurs un nombre croissant de personnes exerçant une activité artistique rémunératrice se déclarent à la Maison des artistes, qu'il s'agisse de leur activité principale ou d'une activité secondaire. Parmi eux, la proportion de personnes tirant de leur activité artistique des rémunérations faibles, voire très faibles, s'est accrue. Ces facteurs externes, liés au progrès du recensement des artistes, ne permettent pas de tirer de conclusions quant à une paupérisation des assujettis. En effet, les données ne permettent pas de déterminer la part que représentent les revenus artistiques dans l'ensemble des revenus des assujettis.

Source : Maison des artistes/DEPS, 2011

\section{RÉSUMÉ}

En 2009, la Maison des artistes affilie 24000 artistes auteurs, principalement graphistes et peintres. Leur nombre s'est élevé en moyenne de $6 \%$ par an entre 1999 et 2006 et est relativement stable depuis 2007.

Près de $60 \%$ des artistes auteurs affiliés sont des hommes. Toutefois, les femmes sont de plus en plus nombreuses, en particulier parmi les plasticiens, les peintres et les graveurs. Les artistes auteurs sont âgés en moyenne de 44 ans et vivent pour plus de la moitié en Île-de-France.

La moitié se partage $15 \%$ de l'ensemble des revenus d'artistes auteurs affiliés à la Maison des artistes, tandis que les $10 \%$ d'artistes auteurs les mieux rémunérés concentrent à eux seuls $43 \%$ de ces revenus.

\section{ABSTRACT}

In 2009, the Maison des Artistes had a membership of 24,000 artist-authors, mainly graphic designers and painters. Their numbers increased by an average of 6\% per annum between 1999 and 2006, and has remained relatively stable since 2007.

Almost $60 \%$ of artist-author members are men. However, the proportion of women is increasing, particularly amongst visual artists, painters and engravers. The average age of artist-author members is 44 , with the majority of them living in the Ile de France area.

Around 50\% of them share in 15\% of the Maison des Artistes' artist-author revenue, whilst some $10 \%$ of the wealthier artist-authors are responsible for $43 \%$ of the Maison's revenue. 

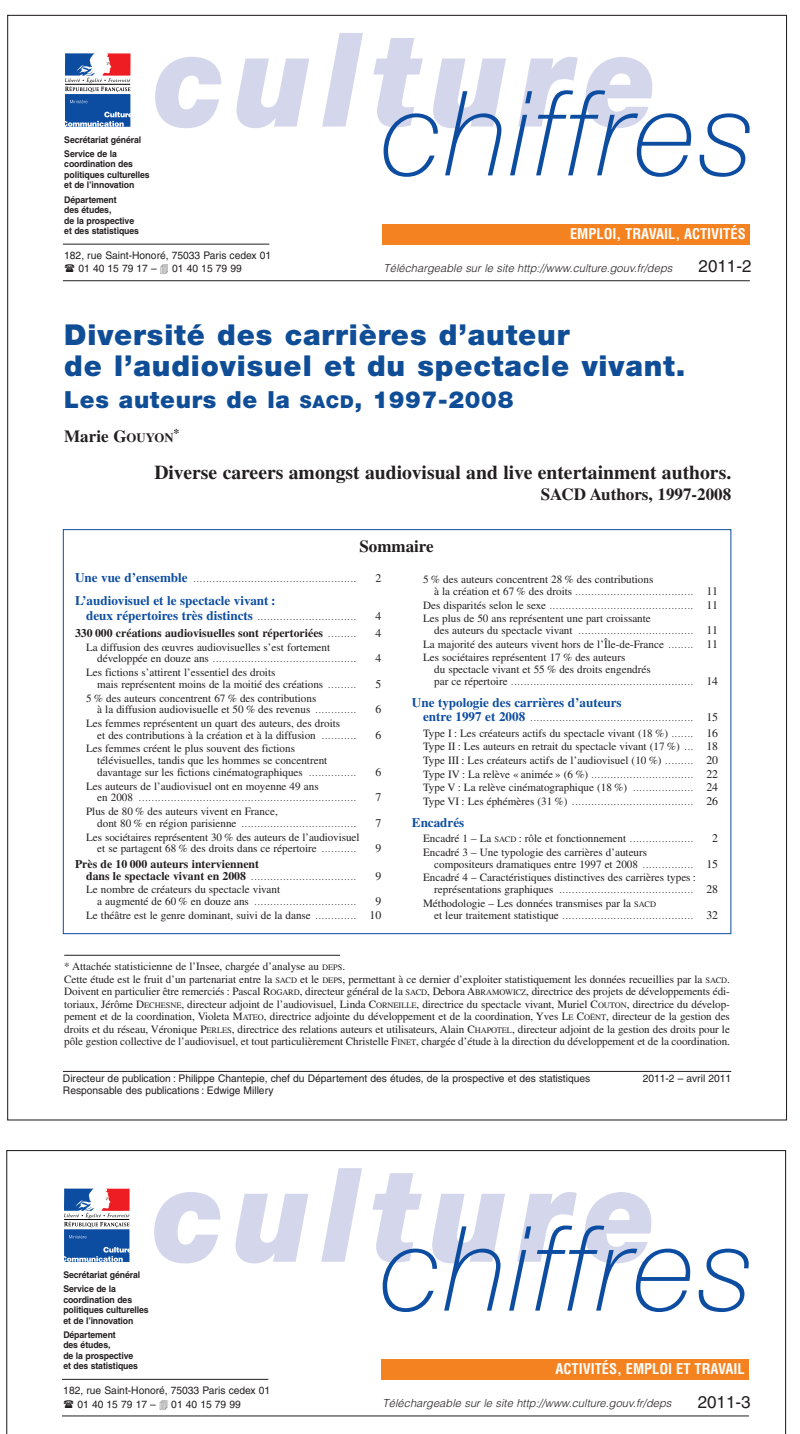

Écrivains, photographes, compositeurs... Les artistes auteurs affiliés à l'Agessa en 2008* Marie Gouyon**

Writers, photographers, composers...

De plus en plus d'artistes auteurs affiliés

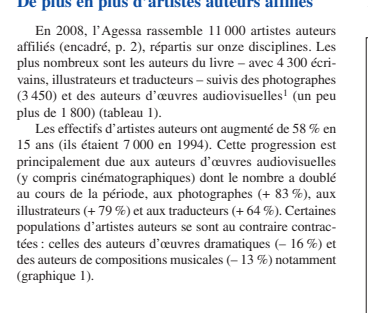

Une population qui se féminise, inégalement selon les disciplines

En 2008, deux artistes auteurs sur trois sont des hommes. Cette surreprésentation masculine est nettement
plus marquué que dans I'ensemble de la population active
en emploi, composié a a $53 \%$ d'hommes?

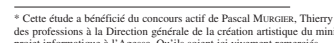

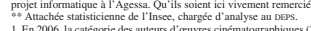

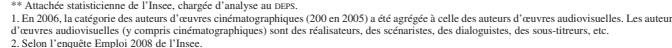

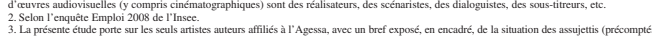

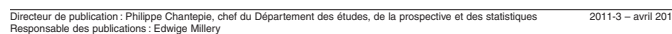

\section{Marie Gouyon}

\section{Diversité des carrières d'auteur de l'audiovisuel et du spectacle vivant. Les auteurs de la SACD, 1997-2008}

Paris, DEPS, Ministère de la Culture et de la Communication, coll. «Culture chiffres »,

2011-2, avril 2011

En 2008, la Société des auteurs et compositeurs dramatiques (SACD) a versé 136 millions d'euros de droits à 17700 auteurs et compositeurs dramatiques. Entre 1997 et 2008, les répertoires gérés par la SACD ont beaucoup évolué, en particulier celui de l'audiovisuel, du fait des mutations technologiques intervenues depuis la fin des années 1990 dans ce secteur: apparition puis généralisation de la télévision numérique terrestre (TNT), développement des chaînes thématiques, etc.

Les auteurs se distinguent nettement les uns des autres selon le répertoire (audiovisuel ou spectacle vivant) auquel ils participent, leur durée et leur volume de production, leur niveau de contributions aux diffusions et aux représentations, ainsi par les montants de droits perçus. La population des auteurs et compositeurs dramatiques reste âgée et très masculine, bien que les femmes y soient de plus en plus nombreuses.

\section{Marie Gouron \\ Écrivains, photographes, compositeurs... Les artistes auteurs affiliés
à l'Agessa en 2008}

Paris, DEPS, Ministère de la Culture et de la Communication, coll. "Culture chiffres », 2011-3, avril 2011

En 2008, l'Association pour la gestion de la sécurité sociale des auteurs (Agessa) affilie 11000 artistes auteurs, principalement du livre, de la photographie et de l'audiovisuel. Leur nombre s'est élevé de $58 \%$ au cours des quinze dernières années.

Les deux tiers des artistes auteurs affiliés sont des hommes. Toutefois, les femmes sont de plus en plus nombreuses, en particulier parmi les écrivains, les photographes et les auteurs d'œuvres audiovisuelles et dramatiques. La population des artistes auteurs est assez âgée (49 ans en moyenne) et fortement implantée en Île-de-France.

La moitié se partage moins de $10 \%$ de l'ensemble des revenus d'auteurs affiliés à l'Agessa, tandis que les $10 \%$ d'artistes auteurs les mieux rémunérés concentrent à eux seuls plus de la moitié de ces revenus.

Tous les documents publiés par le DEPS sont téléchargeables sur http://www.culture.gouv.fr/deps Le DEPS n'assurant pas de diffusion physique de ces documents, nous vous proposons de vous informer régulièrement des parutions par message électronique. Pour ce faire, merci de bien vouloir nous communiquer votre courriel à l'adresse contact.deps@culture.gouv.fr 